\title{
Sparsity and level set regularization for near-eld electromagnetic imaging in 3D
}

DOI:

10.1088/1361-6420/ab44ed

Document Version

Accepted author manuscript

Link to publication record in Manchester Research Explorer

\section{Citation for published version (APA):}

Hiles, A., \& Dorn, O. (2020). Sparsity and level set regularization for near-eld electromagnetic imaging in 3D. Inverse Problems, 36(2), [025012]. https://doi.org/10.1088/1361-6420/ab44ed

\section{Published in:}

Inverse Problems

\section{Citing this paper}

Please note that where the full-text provided on Manchester Research Explorer is the Author Accepted Manuscript or Proof version this may differ from the final Published version. If citing, it is advised that you check and use the publisher's definitive version.

\section{General rights}

Copyright and moral rights for the publications made accessible in the Research Explorer are retained by the authors and/or other copyright owners and it is a condition of accessing publications that users recognise and abide by the legal requirements associated with these rights.

\section{Takedown policy}

If you believe that this document breaches copyright please refer to the University of Manchester's Takedown Procedures [http://man.ac.uk/04Y6Bo] or contact uml.scholarlycommunications@manchester.ac.uk providing relevant details, so we can investigate your claim.

\section{OPEN ACCESS}




\title{
Sparsity and level set regularization for near-field electromagnetic imaging in $3 \mathrm{D}$
}

\author{
A J Hiles ${ }^{a}$ and O Dorn ${ }^{a}$ \\ a The University of Manchester, Alan Turing Building, Oxford Road, Manchester, \\ M13 9PL, UK \\ E-mail: alex.hiles@manchester.ac.uk \\ E-mail: oliver.dorn@manchester.ac.uk
}

September $2019 \ddagger$

\begin{abstract}
In this paper, we propose and compare two novel reconstruction strategies for near-field electromagnetic imaging of regions in 3D surrounded by walls or shields. Our focus is on the estimation of electrical conductivity profiles inside regions which are roughly equivalent in size to small rooms or medium-sized containers, from electromagnetic data obtained at one given frequency. This setup has interesting applications in the surveillance of activities behind walls, the screening of boxes or containers at ports or airports, or the monitoring of processes inside regions which might contain hazardous materials. Moreover, the techniques proposed here can easily be adjusted to imaging situations at larger or smaller scale; as often found in geophysical or non-destructive testing applications. The two novel regularization techniques proposed here are based on a sparsity promoting regularization scheme on the one hand, and a level set based shape evolution technique on the other. In our numerical simulations, we perform $3 \mathrm{D}$ reconstructions from noisy simulated data and compare the results with those obtained from a standard $L^{2}$-type reconstruction approach. Our results suggest, in the applications considered here, that the two proposed novel schemes are potentially able to yield significantly improved reconstructions compared to more traditional techniques.
\end{abstract}

\section{Introduction}

Near-field electromagnetic (EM) imaging is a well-known imaging technology which has not received as much attention in the past as many other alternative electromagnetic techniques. These alternatives include, for example, ground-penetrating radar, synthetic aperture radar, X-ray tomography and microwave imaging, amongst others [44]. The use of relatively low frequencies in near-field imaging makes it more difficult to obtain high resolution reconstructions, and the design of efficient antennas is challenging. Moreover,

‡ CBritish Crown Owned Copyright 2019/AWE.

This is the Accepted Manuscript version of an article accepted for publication in Inverse Problems. IOP Publishing Ltd is not responsible for any errors or omissions in this version of the manuscript or any version derived from it. The Version of Record is available online at the journal webpage. 
the corresponding imaging task usually requires to employ Maxwell's equations in 3D for the forward modelling; which for a long time was severely limited by the capabilities of standard computer systems. However, over the years a number of very interesting practical applications have evolved where the use of relatively low frequencies comes with great advantages, such as: increased penetration depth through materials, a reduced risk of multi-pathing, and avoidance of certain local minima when solving the underlying optimization problem. These applications include geophysical imaging $[1,18,19,28,46,51,60]$, near field sensing and tracking $[53,58]$, the non-destructive testing of materials $[15,41,59]$, and near-field electromagnetic holography $[2,57]$.

The ongoing interest in those and other fields is continuously refuelled by improvements in computer technology and progress in the construction of efficient antenna systems, which addresses some of the above mentioned difficulties. However, it still remains challenging to design efficient tailor-made reconstruction algorithms which are able to convert the measured electromagnetic signals into useful 3D images, for classical or new applications of this technology. Typically, these images show electromagnetic properties of the investigated materials or make use of them for the localization or tracking of objects.

This paper addresses the challenge above by proposing novel reconstruction algorithms for low frequency near-field electromagnetic imaging; which can potentially be applied at various length scales. We will make use of recently developed novel imaging technologies in order to address the increased ill-posedness of the underlying inverse problem when lowering the probing frequencies. In further detail, we will propose two new reconstruction algorithms, one being based on sparsity regularization and the other one on level set based shape evolution, and compare their performances with a more traditional voxel-based reconstruction scheme which employs standard Tikhonov Philips regularization. We will show that both of these novel approaches have the potential to provide significantly improved reconstructions over voxel based imaging schemes in situations where sharp interfaces and significant contrasts between materials are present in the domain of interest.

Sparsity promoting regularization has received worthy attention in recent years as an alternative to $L^{2}$-based minimization schemes. The technique has been applied very successfully to linear inverse problems $[2,5,6,17,25]$, but results on largescale non-linear inverse problems are still scarce. We refer to the recent publications $[33,34,37,50]$ for a review. In the present paper we will consider such a large-scale nonlinear inverse problem which poses specific challenges as outlined further below. The reason for considering sparsity regularization is the observation that standard TikhonovPhilips regularized $L^{2}$ - based schemes tend to provide over-smoothed reconstructions from low-frequency electromagnetic data, even when the true profiles contain shapes with sharp boundaries. Sparsity promoting regularization schemes have recently been demonstrated to mitigate this smoothing tendency by providing more compact shapes against an approximately known background [34, 37, 50]. Level set regularized reconstruction schemes, on the other hand, are designed explicitly to deliver compact 
shapes with sharp boundaries against the background profile. Depending on the chosen level set representation, the internal profile can be constant or smoothly varying $[9,22,23,24,32,39,40]$. Although more established than sparsity promoting schemes, level set methods still pose many challenges for large scale non-linear inverse problems, including the one addressed in this paper.

In this paper, we formulate both sparsity and level set based regularization schemes for a new and highly challenging imaging situation where a 3D Maxwell model is needed as a forward solver. Even though the numerical experiments demonstrated here have been chosen for imaging small rooms or medium-sized containers with shielded walls or obstacles [53, 58], the same techniques can be applied to the imaging at different length scales. These include, for example, geophysical applications or the non-destructive testing of small specimens $[18,21]$. Notice that for many of those applications the use of higher frequencies (and often employment of only far-field data) is not feasible due to the strong attenuation of the corresponding electromagnetic fields by the walls or surrounding environment. Often, nature of the content prevents use of alternative screening techniques such as X-rays or other high-frequency electromagnetic fields, due to safety restrictions or the potentially damaging effect of such high energy probing fields. In addition to the well-known and well-established geophysical applications of such near-field exploration and monitoring tools $[1,18,19,28,46,51,60]$, we want to highlight here several interesting and very promising new experimental verifications of low frequency screening and imaging applications at the scale of smaller boxes as reported for example in $[14,15,16,59]$. In those works, the goal is to screen metallic containers for specific threats where only extremely low electromagnetic frequencies provide sufficient skin-depth for penetrating the present metallic shields. Novel imaging technologies are required in such applications for making optimal use of the obtained data.

The remaining part of the paper is organized as follows: First, in Section 2 we describe in more detail the setup of our low frequency electromagnetic imaging problem using Maxwell's Equations. Then, Section 3 formulates the general underlying mathematical inverse problem for our three reconstruction schemes. Section 4 introduces the Landweber-Kaczmarz approach which we employ to solve this general inverse problem. Section 5 then introduces an $L^{2}$-based reconstruction scheme, which we use here as a comparison to the other developed strategies. Section 6 formulates the novel sparsity promoting reconstruction scheme, and Section 7 describes the novel level set evolution method. Section 8 first introduces the computational setup to test our three reconstruction schemes introduced in the previous three sections, and then describes the results from our computational experiments. In Section 9 we provide a summary of our work and closing thoughts on our findings. In Appendix A and B, finally, we describe two new and highly efficient line search strategies for the various Kaczmarz type reconstruction schemes developed in this paper which, even though slightly technical, form an essential part of all three numerical algorithms presented here. 


\section{The Near Field Electromagnetic Imaging Problem}

We model the propagation of electromagnetic fields by Maxwell's equations in the frequency domain. Hence

$$
\begin{aligned}
& \nabla \times \mathbf{E}_{j}(\mathbf{x})-a(\mathbf{x}) \mathbf{H}_{j}(\mathbf{x})=\mathbf{M}_{j}(\mathbf{x}) \\
& \nabla \times \mathbf{H}_{j}(\mathbf{x})-b(\mathbf{x}) \mathbf{E}_{j}(\mathbf{x})=\mathbf{J}_{j}(\mathbf{x})
\end{aligned}
$$

where we assume a time-dependence $\exp (-i \omega t)$ for a frequency $\omega=2 \pi f$. In equations $(1 a, 1 b), \mathbf{E}$ denotes the electric field, $\mathbf{H}$ the magnetic field, $\mathbf{M}$ the magnetic source and $\mathbf{J}$ the electric source. The material parameters are

$$
a(\mathbf{x})=i \omega \mu(\mathbf{x}) \quad b(\mathbf{x})=\sigma(\mathbf{x})-i \omega \epsilon(\mathbf{x})
$$

where $\sigma(\mathbf{x})$ is the electrical conductivity, $\mu(\mathbf{x})$ is the magnetic permeability, $\epsilon(\mathbf{x})$ is the electrical permittivity.

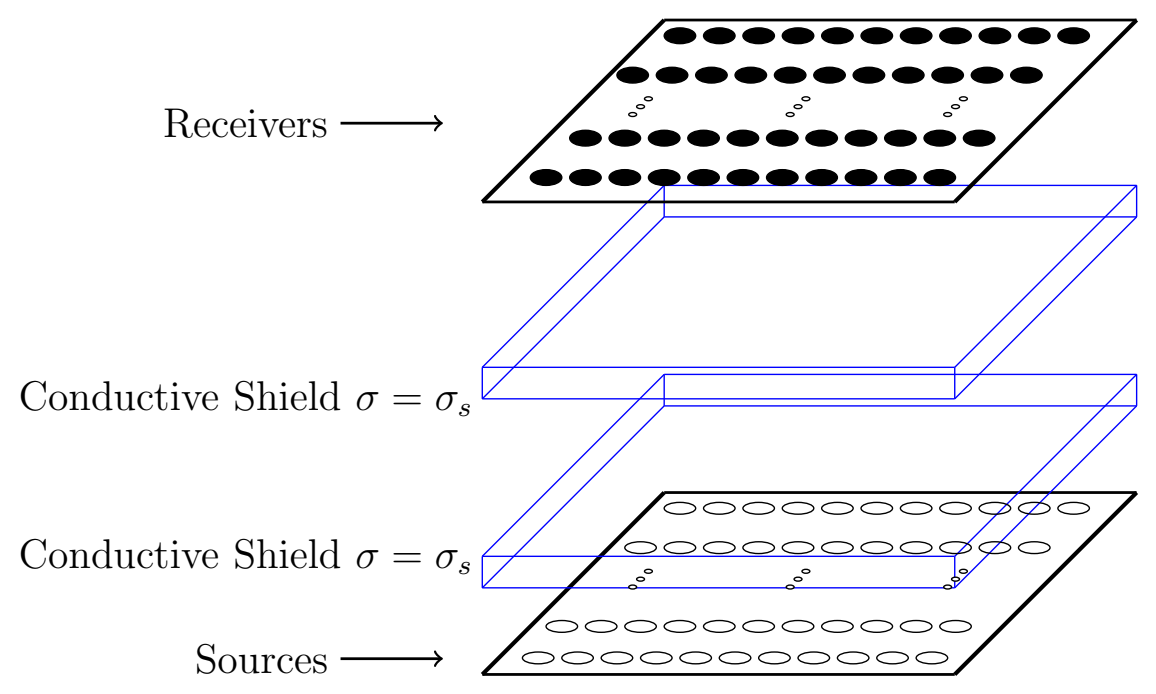

Figure 1. Opposing arrays of Sources and Receivers with $x-y$ conductive shielding. Notice that sources and receivers are only located on top and bottom of the box, but the shielding is applied to all six sides.

A possible experimental setup of the situation described above, and adopted in our numerical experiments, is outlined in Figure 1. Here, the domain of interest is represented by a cuboid-shaped domain $\Omega \subset \mathbb{R}^{3}$ surrounded by shielded walls. We have in total $n_{s}$ different applied source distributions, where the index $j$ specifies that the $j$ th source distribution is considered, $j=1, \cdots, n_{s}$. We model the sources as rectangular wire loops positioned just outside the shielding at two sides of the rectangular box. These give rise to probing fields $\mathbf{E}_{j}$ and $\mathbf{H}_{j}$ in $(1 a, 1 b)$, which are then measured at our receiver locations. For better visualization, we display in Figure 1 only one set of the three opposing conductive shields (i.e. completely surrounding the region of interest), mentioning that similar shields are also present at the four additional sides of the box. The sources and receivers, however, are only located at the two opposed walls towards the $z$ direction. There are no sources or receivers at the other four sides of the box. 
Notice that the shielding complicates the solution of the inverse problem described below due to their attenuation of electromagnetic signals, the so-called skin-effect.

In the inverse problem considered here, we are interested in estimating the conductivity $\sigma(\mathbf{x})$ inside this domain of interest from data measured outside of it. For simplicity, whilst also addressing a proof of concept scenario, we choose $\mu(\mathbf{x})=\mu_{0}$ and $\epsilon(\mathbf{x})=\epsilon_{0}$. This means two of the three material parameters are constant and known throughout the entire domain. This assumption will be relaxed in our future research. Moreover, we assume some reasonable prior knowledge on the background conductivity profile; here being that shielded walls surround a region of interest filled with air. Embedded in this region of interest are inclusion(s) with unknown contrast, topology and shapes. These inclusions need to be reconstructed from few electromagnetic data at one single frequency. Therefore, the zero subscript in $\mu_{0}$ and $\epsilon_{0}$ indicates that free space values are taken with respect to the given material parameters. In addition, we assign a very low value $\sigma \approx 10^{-8} \mathrm{~S} / \mathrm{m}$ to the background representing the conductivity of air. The shielded walls, on the other hand, are here assumed to have a known topology and a moderate conductivity value of $\sigma \approx 0.1 \mathrm{~S} / \mathrm{m}$. In future work also such an assumption will be relaxed. As mentioned, the conductivity values of the inclusions are assumed to be higher than the background (here $\sigma \approx 0.5 \mathrm{~S} / \mathrm{m}$ ), but its value is unknown in the inverse problem and needs to be reconstructed together with their shapes and topology from the data. At present we assume that all inclusions share the same (unknown) internal conductivity value, but this assumption will also be relaxed in our future work. The values used in our numerical experiments are summarized in Table 1.

Table 1. Conductivity parameters used in numerical experiments

\begin{tabular}{|c|c|}
\hline & $\sigma\left(\mathrm{Sm}^{-1}\right)$ \\
\hline Background & $1 \times 10^{-8}$ \\
Unknown Inclusions & 0.5 \\
Shields & 0.1 \\
\hline
\end{tabular}

\section{Mathematical formulation of the inverse problem}

Inverse problems for Maxwell's equations have been investigated for a long time in a variety of applications and settings. For recent overviews of available theoretical and computational results we refer to $[12,13,60]$ and the references provided there. The important questions of uniqueness and differentiability of related inverse problems are addressed for example in $[8,11,31,56]$. For derivation of the algorithms in this paper, we assume differentiability (in properly chosen function spaces) of all forward mappings that arise here, allowing us to adopt well-established expressions for the relevant derivatives (or sensitivities) with respect to the unknown medium parameters [19, 20, 43, 55, 60].

To start with, we will formulate the inverse problem for the estimation of $\sigma(\mathbf{x})$; which is the main parameter of interest in our work. Since we are formulating three 
different reconstruction schemes, this section will outline the inverse problem in a setting which is consistent to all three. For further use in our Kaczmarz type approach described below, the forward operator is indexed by the source index $j$, which gives rise to the notation $\mathcal{F}_{j}[b]=\mathcal{M}_{j} \mathbf{E}_{j}(\mathbf{x} ; b)$. Here $\mathbf{E}_{j}, \mathbf{H}_{j}$ solve $(1 a, 1 b)$ with parameter $b$ and $\mathcal{M}_{j}$; where the latter is a linear measurement operator which may depend on the source position. In our setup, we take

$$
\mathcal{M}_{j} \mathbf{E}_{j}=\oint_{S_{j}} \mathbf{E}_{j} \cdot \tau d l
$$

where $\mathbf{E}_{j} \cdot \tau$ is the tangential component of $\mathbf{E}$ with respect to the (closed) wire loop $S_{j}$ which acts as a receiver; those we also model to be rectangular loops. Notice that we can simulate for any conductivity distribution $b$ the corresponding predicted measurements $\mathcal{F}_{j}[b]$ and compare them to the physically measured data $\tilde{\mathcal{F}}_{j}$. This yields the residuals $\mathcal{R}_{j}[b]=\mathcal{F}_{j}[b]-\tilde{\mathcal{F}}_{j}$.

Let us denote by $P$ the space of parameters $\mathbf{b}$ (which are assumed here for simplicity to be isotropic together with $\mu_{0}$ and $\left.\epsilon_{0}\right), Z_{j}$ the space of measurements $D_{j}$ and noiseless residuals $\mathcal{R}_{j}, U$ the spaces of states $\mathbf{u}$ and $Y$ the space of sources. In the derivation of our algorithms, we assume that these function spaces are represented by Hilbert spaces consisting of $L^{2}$ - functions which carry a special physical significance. All these function spaces are then equipped with standard inner products in $L^{2}$. In more detail, we choose

$$
\begin{array}{rlrl}
P & :=\mathcal{L}^{2}(\Omega) ; & U:=\left\{\left(\begin{array}{c}
\mathbf{E}(\boldsymbol{x}) \\
\mathbf{H}(\boldsymbol{x})
\end{array}\right), \mathbf{E}, \mathbf{H} \in\left[\mathcal{L}^{2}(\Omega)\right]^{3}\right\} ; \\
Z_{j}:=\left\{\left(\begin{array}{c}
\eta \\
\xi
\end{array}\right), \eta, \xi \in\left(\mathbb{C}^{3}\right)^{n_{s, j}}\right\} ; & Y:=\left\{\left(\begin{array}{c}
\mathbf{J}(\boldsymbol{x}) \\
\boldsymbol{M}(\boldsymbol{x})
\end{array}\right), \mathbf{J}, \boldsymbol{M} \in\left[\mathcal{L}^{2}(\Omega)\right]^{3}\right\} .
\end{array}
$$

We can describe the inverse problem for all three approaches as follows. Assume that we have $n_{s}$ source distributions $\mathbf{q}_{j}\left(j=1,2, \cdots, n_{s}\right)$ located at positions $\mathbf{s}_{j}$ (which are here modelled as small single wire loops of current intensity $I_{j}$ extended over one or more numerical grid cells and with appropriate orientations) such that radiation from these sources is propagated by the Maxwell model in $(1 a, 1 b)$. Furthermore, $n_{r}$ receivers are located at $\mathbf{r}_{j k}\left(k=1,2, \cdots, n_{r}\right)$. Then, given measurements of the EM field $\mathbf{E}_{j}$ at $\mathbf{r}_{j k}$, find the unknown conductivity $\sigma=R e(b)$ (where the symbol Re indicates the 'real part') in $\Omega$ with $b \in P$. Depending on the approach taken, this general model will be refined further below.

One standard way of addressing this general problem is to write down a least squares data misfit functional in terms of residual operators. In our case this reads as

$$
\mathcal{J}[b]=\sum_{j=1}^{n_{s}} \mathcal{J}_{j}[b] ; \quad \mathcal{J}_{j}[b]:=\frac{1}{2}\left\|\mathcal{R}_{j}[b]\right\|_{Z_{j}}^{2} .
$$

Therefore, the residual operator quantifies the mismatch between model prediction (forward problem) and true data. The cost functional $\mathcal{J}[b]$ is useful for monitoring progress of any iterative estimation technique and for the design of suitable reconstruction schemes. For example, we can formulate an optimization problem 
incorporating $\mathcal{J}[b]$ with or without additional suitably chosen regularization terms. Many popular minimization schemes use the formal gradient of (5) for defining search directions:

$$
\nabla \mathcal{J}[b]=\sum_{j=1}^{n_{s}} \nabla \mathcal{J}_{j}[b],
$$

where the individual terms $\nabla \mathcal{J}_{j}[b]$ can be calculated efficiently by a so-called adjoint scheme $[19,20]$. However, note that even when employing an adjoint scheme, for typical values of $n_{s}$ the calculation of (6) in each iteration is still too time consuming when addressing large scale problems. Therefore, in the following we will outline a form of a single-step adjoint scheme which is employed in our calculations.

Assuming sufficient regularity of $R_{j}[b]$ in the chosen function spaces $P$ and $Z_{j}$, let us denote the linearized residual operator by $\mathcal{R}_{j}^{\prime}[b]$ and its adjoint operator with respect to these function spaces by $\mathcal{R}_{j}^{\prime}[b]^{*}$. Let us choose some data $\xi_{j}=\mathcal{R}_{j}[b] \in Z_{j}, b \in P$ and let $\left(\mathcal{E}_{j}, \mathcal{H}_{j}\right)^{T}$ be the solution of the adjoint Maxwell problem:

$$
\left(\begin{array}{cc}
-b & \nabla \times \\
\nabla \times & a
\end{array}\right)\left(\begin{array}{c}
\mathcal{E}_{j} \\
\mathcal{H}_{j}
\end{array}\right)=\left(\begin{array}{c}
\mathbf{0} \\
\mathcal{M}_{j}^{*} \overline{\mathcal{R}_{j}[b]}
\end{array}\right),
$$

where the overline denotes 'complex conjugate' and $\mathcal{M}_{j}^{*}$ is the formal adjoint of the measurement operator. Its application amounts to putting the residuals as artificial adjoint sources at the receiver locations. Then,

$$
\nabla \mathcal{J}_{j}[b]=\mathcal{R}_{j}^{\prime}[b]^{*} \xi_{j}=\mathcal{R}_{j}^{\prime}[b]^{*} \mathcal{R}_{j}[b]
$$

is given by

$$
\left[\mathcal{R}_{j}^{\prime}[b]^{*} \mathcal{R}_{j}[b]\right](\mathbf{x})=\overline{\mathbf{E}_{j}(\mathbf{x}) \cdot \mathcal{E}_{j}(\mathbf{x})} .
$$

The interpretation of $\mathcal{R}_{j}[b]$ as an artificial adjoint source, upon which $\mathcal{R}_{j}^{\prime}[b]^{*}$ is acting on in (8), gives rise to the interpretation of 'back-projecting' the residual data to their (secondary) source. For simplifying the notation below, we implicitly assume that the unknown parameter only consists of the real part of $b$, i.e. whenever we refer to that parameter only, we take the real part of the expression such as that in (8). This assumption holds throughout this paper. For more details on this adjoint scheme we refer to $[19,20,43,55,60]$.

\section{The Landweber-Kaczmarz type approach}

The non-linear problem described here is of large scale in 3D. Usually, iterative techniques are employed for the solution, requiring repeated calculation of descent directions to $\mathcal{J}[b]$ in (5). Most standard schemes require calculation of the full gradient $\nabla \mathcal{J}[b]$ in (6). Depending on the available Maxwell solver, this might consume considerable resources and processing time in each iteration. Therefore, alternative techniques have been developed in order to speed up the inversion process. One of those is the non-linear Landweber-Kaczmarz scheme which is employed here. The technique 
cycles over individual source positions in a certain order and only requires to calculate gradients of partial data sets in each step, which can be done efficiently with the so-called adjoint method as outlined above.

With this scheme it is no longer the primary goal to directly reduce the data misfit to $\mathcal{J}[b]$ in (5) in each individual step. Instead, in each iteration, we reduce the data misfit $\mathcal{J}_{j}[b]$ with respect to the $j$ th source only. For more information regarding this general scheme we refer to $[19,35,36,38,42,44]$.

In more detail, we use the single-step update formula

$$
b_{k+1}=b_{k}-\tau_{k} \nabla \mathcal{J}_{[k]}\left[b_{k}\right],
$$

where we follow, for simplicity, a sequential rule $[k]:=\left(k\right.$ modulo $\left.n_{s}+1\right) \in\left\{1,2, \cdots, n_{s}\right\}$. Alternative approaches, such as using a random selection of sources, are possible as well. Note that similar 'Stochastic Gradient Methods' have become popular in several large scale data sciences applications, which have attracted a lot of interest recently [7, 27]. Those will be investigated in further detail in our future research.

Notice that so far we have not explicitly incorporated any regularization in our analysis of the problem. One regularization tool, which we will employ, is to use tailor-made function spaces when calculating gradient directions for (5). We recall that the formulation of the adjoint problem defined in Section 3 maps into standard $L^{2}$ spaces. This is sufficient and convenient in general, but for the schemes considered further below it will be beneficial to obtain gradient directions in a subset of smoother functions. In particular, such smooth gradient directions provide greater stability of the reconstruction schemes. In order to derive this alternative form with respect to the parameter $b$, let

$$
W_{1}(\Omega)=\left\{f: f \in L_{2}(\Omega), \nabla f \in L_{2}(\Omega), \frac{\partial f}{\partial n}=0 \text { at } \partial \Omega\right\},
$$

equipped with the weighted inner product

$$
\langle v, w\rangle_{W_{1}(\Omega)}=\alpha\langle v, w\rangle_{L_{2}(\Omega)}+\beta\langle\nabla v \cdot \nabla w\rangle_{L_{2}(\Omega)} .
$$

Here, $\alpha \geq 1$ and $\beta>0$ are suitably chosen regularization parameters. Let us denote the adjoint operator with respect to this new function space by $R_{j}^{\prime}[b]^{s}$, where the superscript $s$ indicates 'smoothed'. It has been shown in $[26]$ that $R_{j}^{\prime}[b]^{s}$ can be obtained from $R_{j}^{\prime}[b]^{*}$ by a simple post-processing step. In more details, we obtain the corresponding smoothed gradient $\nabla \mathcal{J}_{j}^{s}[b]$ (which is based on those adjoints via (7)) by solving

$$
(\alpha I-\beta \Delta) \nabla \mathcal{J}_{j}^{s}[b]=\nabla \mathcal{J}_{j}[b] \text { in } \Omega,
$$

where $I$ and $\Delta$ are the Identity and the Laplacian operator respectively, $\nabla \mathcal{J}_{j}[b]$ is the unsmoothed gradient, and appropriate boundary conditions are applied. We mention that this smoothed gradient is also often referred to as a Sobolev gradient in the literature [45]. For more information on practical ways of calculating this Sobolev gradient, we refer the reader to the more detailed discussion in [26]. 


\section{5. $\mathrm{L}^{2}$-based inversion with a dynamic line search scheme}

Our goal is to iteratively apply updates to the unknown parameter functions following the above derived Sobolev type descent directions, but in a Kaczmarz type approach. Whereas in standard gradient based optimization schemes a line search is performed following well established rules and criteria [47], the task of finding an optimal step size in each step of such Kaczmarz type approaches is not yet well understood. The reason being is that only part of the data is involved, such that large step sizes in this partial gradient direction might severely deteriorate the data fit which has been achieved by previous updates from different parts of the data set. Therefore, one must be careful when choosing $\tau_{k}$ as this parameter dictates how much we reduce the cost with respect to one source only. In Appendix A, we will suggest a new line search strategy which has been proven to work well in our particular application. The complete LK reconstruction algorithm including this dynamic correction line search is described in Algorithm 1.

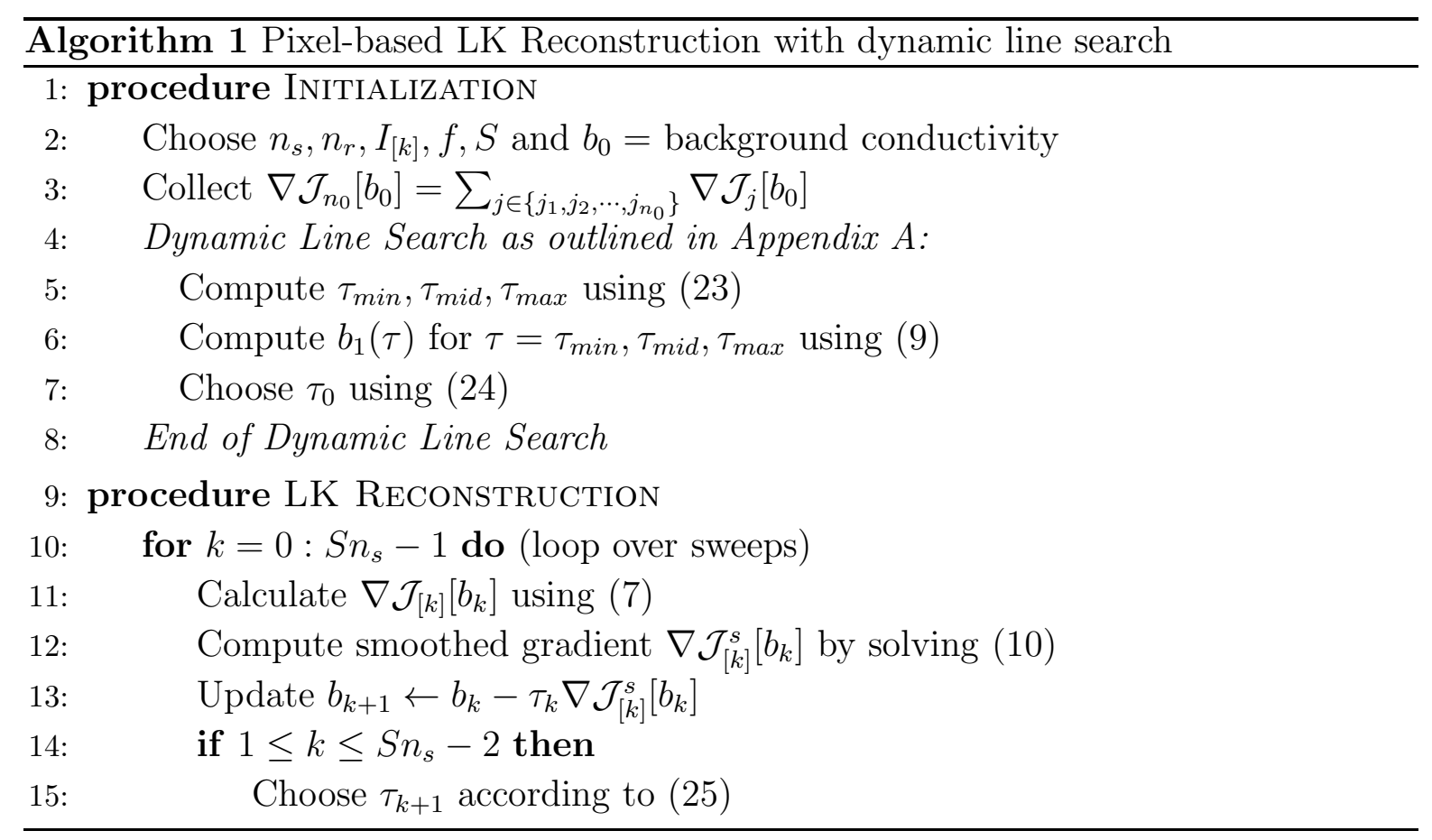

\section{Sparsity Promoting Regularization}

The regularization employed thus far is implicit; in the sense that, the gradient descent of our cost functional is projected to a smoother space. Whereas this helps stabilizing the inversion process, it also tends to deliver quite over-smoothed conductivity profiles. This over-smoothing property of pixel based inversion schemes also has been observed when not using Sobolev gradients but standard $L^{2}$ gradients to (5) for the inversion [19, 20]. In situations where it is known or expected that the unknown part of the conductivity distribution has compact support, this approach therefore does not seem to be optimal. 
Instead, one can try to incorporate this a priori knowledge on expected discontinuities of medium parameters in the domain, enclosing regions of high conductivity and compact support, into the inversion model. Recently, a sparsity promoting regularization scheme has been suggested for similar situations in other non-linear imaging modalities such as electrical impedance tomography or optical tomography, see for example [33, 34, 50]. We will develop in the following such a sparsity promoting scheme for the application of near field electromagnetic imaging in 3D.

In our sparsity promoting regularization scheme we modify our cost functional with the addition of an $l^{1}$ penalty term, such that contributions in the parameter space, which are considered small in a predefined sense, are penalized. Sparsity can be considered in several ways, with respect to a variety of basis systems or over-complete dictionaries. We will consider here sparsity with respect to the standard voxel basis, which favours reconstructions with compact support. Such a process can be computed practically by using so-called shrinkage operators [17].

With the above, we decompose $b=b_{0}+\delta b$ where $b_{0}$ denotes an approximately known background and $\delta b$ the unknown inclusions. We now define a new cost functional

$$
\hat{\mathcal{J}}(b)=\mathcal{J}(b)+\gamma\|\delta b\|_{1}
$$

with the assumption that

$$
\|\delta b\|_{1}=\sum_{k}\left|\left\langle\delta b, \psi_{k}\right\rangle\right|
$$

where in our case $\left\{\psi_{k}\right\}$ is an orthonormal basis, but in other applications it could be an over-complete dictionary.

Due to the $\|\delta b\|_{1}$ term, we see that the cost functional in (11) is no longer differentiable. Following suggestions in $[17,33,34]$ and results shown in [50], we perform the following steps to promote sparsity in the reconstruction:

(i) Compute gradient descent direction $\nabla \mathcal{J}\left[b_{k}\right]$;

(ii) Using (i) calculate $\nabla \mathcal{J}^{s}\left[b_{k}\right]$;

(iii) Apply shrinkage on $\delta b_{k+1}=\delta b_{k}-\tau \nabla \mathcal{J}^{s}\left[b_{k}\right]$.

Explicitly, these three steps can be combined to write

$$
\delta b_{k} \leftarrow S_{\tau_{k} \gamma}\left(\delta b_{k}-\tau_{k} \nabla \mathcal{J}_{[k]}^{s}\left[b_{k}\right]\right)
$$

where

$$
\left(S_{\tau \gamma}(x)\right)_{i}= \begin{cases}\left(\left|x_{i}\right|-\tau \gamma\right) \operatorname{sign}\left(x_{i}\right) & \text { if }\left|x_{i}\right|>\tau \gamma \\ 0 & \text { otherwise }\end{cases}
$$

The sparsity promotion is thus embedded in the shrinkage operator, as this influences how the gradient descent behaves in the next iteration.

Reduction of our new cost functional $\hat{\mathcal{J}}(b)$ is found by performing the steps outlined above. Notice that again finding an efficient line search strategy is essential for the success of the approach. In contrast to the above mentioned previous applications, 
when initializing $\tau_{k}$, we perform the same line search technique as that in the pixelbased, mentioning again that this is outlined in Appendix A. Differing from the pixelbased inversion, however, we now incorporate the thresholding parameter $\Lambda=\tau \gamma$, defined in (14). This parameter controls the intended sparsity level of the conductivity reconstruction. Here, we choose

$$
\Lambda=d_{1} \max \left(\left|\delta b_{1}\right|\right)
$$

where

$$
\delta b_{1}=\delta b_{0}-\tau_{0} \nabla \mathcal{J}_{0}^{s}\left[b_{0}\right]
$$

and $d_{1} \in\left(0, y_{2}\right)$, where $y_{2}$ is typically unity, but other choices are possible. Our assumption on the thresholding term is that the maximum value of $\delta b_{1}$ should have the same order of $\delta b_{k}$ for $k>1$. Furthermore, $d_{1}$ can be interpreted as a parameter that chooses the level of zero filling in the reconstruction. The complete LK-Sparsity reconstruction scheme including a line search for initializing $\tau_{k}$ is presented in Algorithm 2.

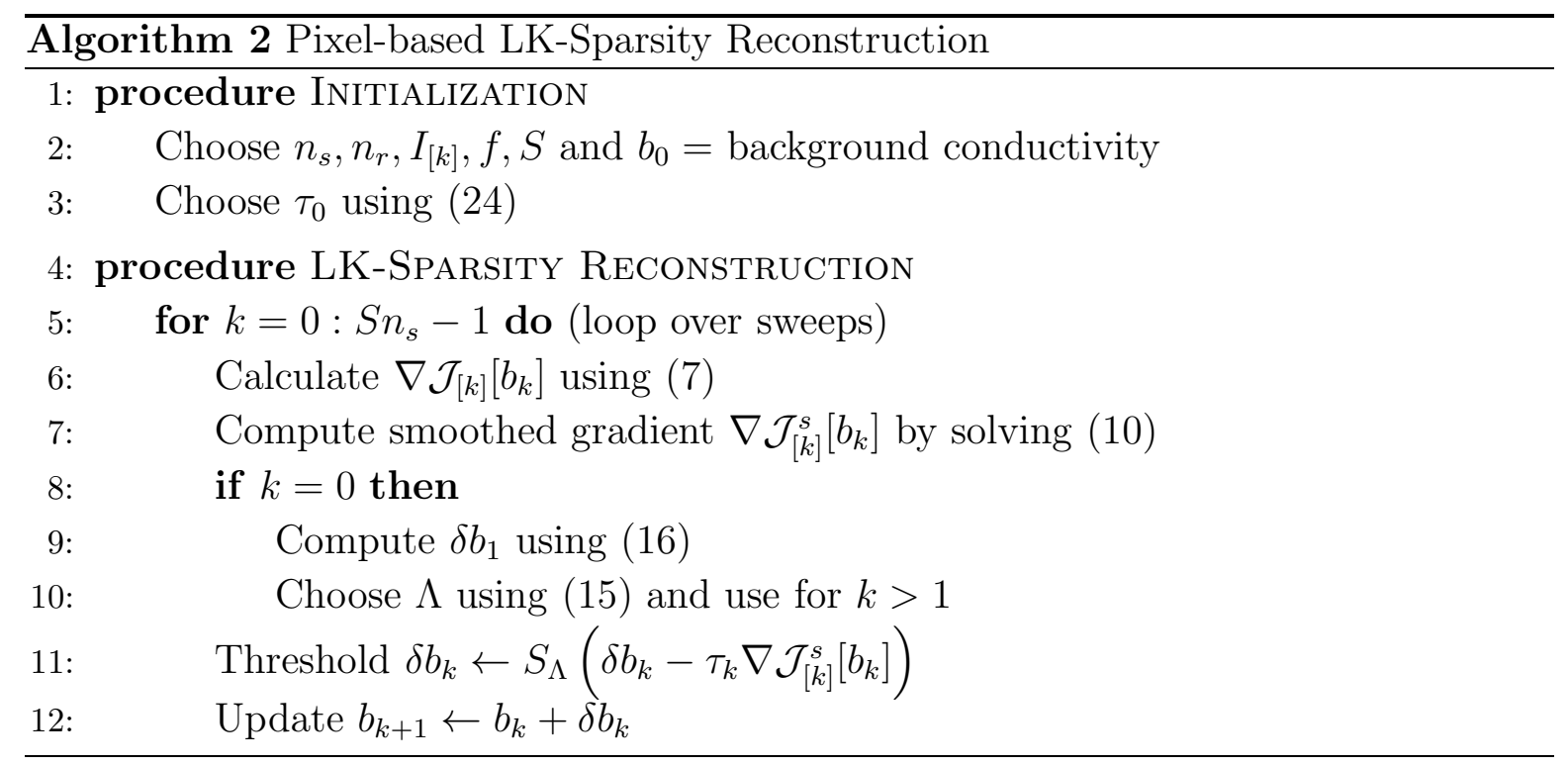

\section{Level Set Shape-based Reconstruction}

The sparsity promoting scheme proposed in the previous section is useful when inclusions with compact support or specific shape embedded in some approximately known background are sought for. A similar effect has been observed in a variety of applications when using a level set based approach which directly uses a shape-based model for the unknown inclusions. Originally, level set methods were developed by Osher and Sethian as a means for tracking a propagation of interfaces in image processing and computational physics $[48,54]$. This led to Santosa applying their ideas in the arena of inverse problems [52]. Since then, level set methods for nonlinear inverse problems 
have been applied across a whole range of imaging modalities, see [10, 22, 23] for recent reviews.

The key difference between the level set shape-based approach compared to the pixel-based reconstructions described in Algorithms 1 and 2 is that some estimate of the true conductivity value is assumed known a priori. This additional information is observed to improve reconstructions of the topology and shapes of the inclusions significantly in such low-frequency electromagnetic problems, even in cases where the actual value of the internal conductivity is significantly over or underestimated by the approximate value plugged into the inversion routine [18]. Therefore, in many practical applications such shape based methods actually provide very useful approaches for detecting and estimating general shapes of inclusions from few data. Moreover, if needed, the estimation of such internal parameter values can be made part of the optimization problem such that an estimate of the internal conductivity value can be corrected throughout the optimization procedure. We will not include such an additional optimization loop in the algorithm proposed here, but instead test the algorithm against incorrectly estimated internal conductivity values. For details, see below.

To discuss the technical implications of such a level set based shape evolution approach, let us begin by introducing a sufficiently smooth level set function $\phi: \Omega \rightarrow \mathbb{R}$, which defines a shape $S$ by

$$
\Pi(\phi)(\mathbf{x})= \begin{cases}b_{i}(\mathbf{x}) & \text { in } \mathrm{S} \text { where } \phi(\mathbf{x}) \leq 0, \\ b_{e}(\mathbf{x}) & \text { in } \Omega \backslash S \text { where } \phi(\mathbf{x})>0,\end{cases}
$$

where the indices $i$ and $e$ denote interior and exterior respectively. The boundary $\partial S$ is given by the zero level set $\partial S=\{\mathbf{x} \in \Omega: \phi(\mathbf{x})=0\}$. This representation can be written in the convenient form

$$
\Pi(\phi)(\mathbf{x})=b_{i}(1-H(\phi))+b_{e} H(\phi)
$$

where $H$ is the Heaviside function. In the level set inversion, we are interested in finding a suitable $\phi$ such that the data misfit is minimized. With this in mind, let

$$
\mathcal{T}(\phi)=\mathcal{R}[\Pi(\phi)], \mathcal{J}(\phi)=\|\mathcal{T}(\phi)\|_{\mathcal{L}^{2}(\Omega)}^{2},
$$

which yields the formal gradient

$$
\nabla \mathcal{J}[\phi]=\sum_{j=1}^{n_{s}} \nabla \mathcal{J}_{j}[\phi] .
$$

From $(7,8)$ we obtain the analog

$$
\nabla \mathcal{J}_{j}[\phi](\mathbf{x})=\mathcal{T}_{j}^{\prime}[\phi]^{*} \mathcal{T}_{j}[\phi](\mathbf{x})=\Pi^{\prime}(\phi) \overline{\mathbf{E}_{j}(\mathbf{x}) \cdot \mathcal{E}_{j}(\mathbf{x})},
$$

where

$$
\Pi^{\prime}(\phi)=\left(b_{e}-b_{i}\right) \delta(\phi)
$$

and $\delta(\cdot)$ is the Dirac Delta distribution. In the advent of numerical experiments, such a distribution has to be approximated by a function in a suitable space. Therefore, we choose a narrowband function $[48,54]$, such that $\delta(\phi) \approx \chi_{B_{d}(\Gamma)}$, where

$$
B_{d}(\Gamma)=\left\{\mathbf{x}:\|\mathbf{x}-\Gamma\|_{2}^{2} \leq d / 2\right\},
$$


$d>0$ and $\chi_{D}$ is the characteristic function. Other approximations are possible as well. In general, we assume that boundaries of objects are smooth. Therefore, as in the $L^{2}$-based LK and LK-Sparsity algorithms, one can map the gradient to a smoother space.

Thus, in spirit of the LK technique, we obtain the following iterative scheme

$$
\phi_{k+1}=\phi_{k}-\tau_{k} \nabla \mathcal{J}_{[k]}^{s}\left[\phi_{k}\right],
$$

where $\tau_{k}$ is a step size to be discussed. It must be stated that the initial level set function is chosen to be a signed distance function [48, 54], representing some arbitrary initial shape. Typically, a level set representation of an ellipsoid or sphere in the centre of $\Omega$ suffices in simple geometries, such as those considered here. Better adapted initial guesses can speed up the reconstruction, though, and for that reason should be used whenever available. Once this initial shape has been assigned, we can proceed with the iterative scheme in (19) for $k \geq 1$.

As in the previous two Kaczmarz based reconstruction schemes, an efficient line search is vital for success in this shape based scheme. It turns out that for shape based schemes a very simple yet efficient line search can be designed which does not require any additional forward solves. An improvement of the line search scheme introduced recently in [21] will be presented in Appendix B and is used in Algorithm 3.

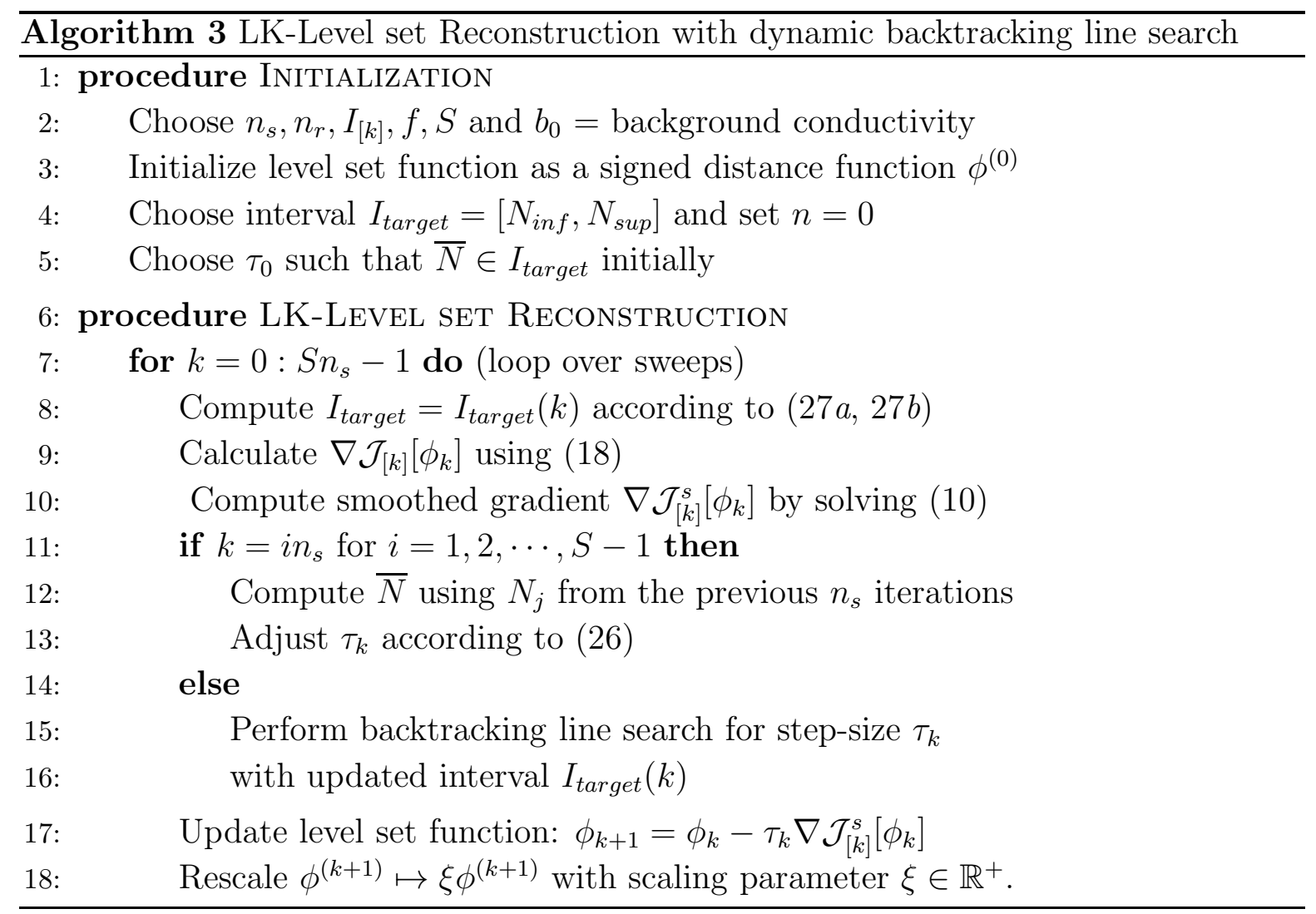




\section{Numerical Experiments}

\subsection{Computational Setup}

We model a cube-shaped container by a domain $\Omega^{h}=[3] \times[3] \times[3] \mathrm{m}^{3}$. The domain $\Omega^{h}$ is divided into [20] $\times[20] \times[20]$ uniform rectangular grid cells, each of them having dimension $[0.15] \times[0.15] \times[0.15] \mathrm{m}^{3}$. For practical reasons, sources and receivers are located along two planes with constant $z$ coordinates, opposing each other at both sides of the box (See Figure 1). There are no sources or receivers facing the other four sides of the domain. As already mentioned, we equip $\Omega^{h}$ with a homogeneous background conductivity, shielded walls on all six sides, and some unknown embedded internal object(s). The shielded walls have dimensions $[3] \times[3] \times[0.30] \mathrm{m}^{3}$ on the two sides parallel to the $x y$ plane, and the same but shifted dimensions for the corresponding coordinates at the other four sides. These shielded walls are composed of material with conductivity $\sigma_{s}=0.1 \mathrm{Sm}^{-1}$. In our numerical experiments, we use two distinct test phantoms; the first consists of two rectangular small cuboid inclusions embedded in the interior of the domain of interest, and the second is a horizontal torus shaped inclusion. We choose both phantoms to be composed of material with conductivity $\sigma_{t}=0.5 \mathrm{Sm}^{-1}$. The remaining space (not occupied by those inclusions) is filled with air, which is assumed here to have a homogeneous background conductivity $\sigma_{0}=1 \times 10^{-8} \mathrm{Sm}^{-1}$. A summary of the used conductivity values has been shown in Table 1 in Section 2. Furthermore, a single frequency $f=1 \mathrm{MHz}$ is used. We consider $\left(n_{s}, n_{r}\right)=(16,100)$, where the distribution of these sources and receivers follow that in Figure 1. Each source has dimension $[0.60] \times[0.60] \times[0.15] \mathrm{m}^{3}$ and each receiver has dimension $[0.15] \times[0.15] \times[0.15] \mathrm{m}^{3}$. As described in Section 3, we excite each source (electric wire loop) with an electric current $I_{j}=I=0.1 \mathrm{~A}$.

Our discretization of the Maxwell model in $(1 a, 1 b)$ overall follows the scheme described in $[3,4,29,30]$ and is implemented by using the programming language Python. The scheme discretizes a vector potential formulation of $(1 a, 1 b)$ and integrates over finite volumes to find a discrete Maxwell system. We then solve the discrete Maxwell system iteratively using the BiCGSTAB algorithm, with tolerance $10^{-4}$. A simple postprocessing step is then performed to obtain the electromagnetic fields $\mathbf{E}$ and $\mathbf{H}$ from the vector potential.

In all our numerical experiments presented here, we use synthetically generated data using the same forward modelling scheme, but add $1 \%$ white Gaussian noise to the data before its use as data in the inversion process. For future work we plan to use data generated by a different independent forward solver, but in this study aiming at a proof-of-concept analysis we decided to use noisy simulated data instead. Thus, the data $\tilde{\mathcal{F}}_{j}$ admits a decomposition

$$
\tilde{\mathcal{F}}_{j}=\mathcal{M}_{j} \hat{\mathbf{E}}_{j}(\mathbf{x})\left(1+c \epsilon_{j}\right),
$$

where $\hat{\mathbf{E}}_{j}(\mathbf{x})$ is the electric field generated by the test phantom, $\epsilon_{j} \sim \mathcal{N}(0,1)$ and $c \in \mathbb{R}$ is a scaling parameter that dictates the noise level. Across all reconstruction schemes 
for near-field electromagnetic imaging considered here, we assume the computational setup described in this section and that the measured data decomposes as in (20).

The numerical experiments are structured as follows. The two phantoms described above are displayed in Figure 2 (marked there as 'True Phantom') in form of surface plots, both enclosed inside the shielding shell. We have performed all numerical experiments on both phantoms with comparable quality of results, but for the sake of brevity we will display here the results in the following way:

(i) LK-Level set Reconstructions for Phantoms 1 and 2 using Algorithm 3;

(ii) LK-Pixel Reconstructions for Phantom 1 using Algorithm 1;

(iii) LK-Sparsity Reconstructions for Phantom 1 using Algorithm 2.

For the pixel-based reconstructions, we will display cross sections of the recovered conductivity (including the shielding shell). For the level set reconstructions, we will consider both, cross sections and three-dimensional surface plots. Surface plots are available here as the unknown part of the conductivity is binary by definition. Therefore, the recovered conductivity inside the domain of interest admits only two values; the background and target conductivity. When displaying level set reconstructions in form of surface plots, we will not show the shielding shell. For the LK-Sparsity reconstructions, we will display cross sections of the recovered conductivity including the shielding shell. We use the line search criteria for the LK-Sparsity scheme once for each test phantom and use the resultant parameter $\tau_{0}$ for all sparse reconstructions, with respect to each test phantom. Moreover, we will provide the corresponding plots of the evolution of residuals, step sizes $\tau_{k}$ and, if applicable, $\bar{N}$ against the sweep number $s$. We will also provide comparisons of the three methods afterwards.

In more technical aspects, we have chosen in these numerical experiments the intervals $I_{\text {target }}=\left[N_{\text {inf }}, N_{\text {sup }}\right]$ where $N_{\text {inf }}=0$ and $N_{\text {sup }}=25, I_{\text {target }}^{\prime}=\left[\hat{N}_{\text {low }}, \hat{N}_{\text {high }}\right]$ where $\eta=1 / 5, S=200, N_{\text {low }}=\frac{1}{4} N_{\text {sup }}$ and $N_{\text {high }}=\frac{3}{4} N_{\text {sup }}$. These are our parameters for the dynamic intervals described in $(27 a, 27 b)$. Bounds of the admissible interval range $I_{\text {target }}$ are displayed in block black lines and bounds of the desired region $I_{\text {target }}^{\prime}$ are shown with dashed green lines. Across all three inversion schemes, we perform $N=25$ steps of the heat equation for smoothing the $L^{2}$ gradient.

\subsection{Level set reconstruction with correctly chosen contrast value}

Shown in Figure 2 are surface plots of 3D LK-Level set reconstructions for two test phantoms, where the correct internal conductivity value is employed during the reconstruction. By observation, the algorithm has distinguished between two objects within the domain and has recovered parts of the torus. We notice that the method manages to resolve two distinct shapes and a torus shaped object.

Figure 3 shows an evolution of the average number of pixels $\bar{N}$ that change per sweep and its relationship with the step size $\tau_{k}$. In particular, the algorithm attempts to keep $\bar{N} \in I_{\text {target }}^{\prime}(s)$ for each sweep $s$ by adjusting $\tau_{k}$ according to the line search scheme discussed in Appendix B. 


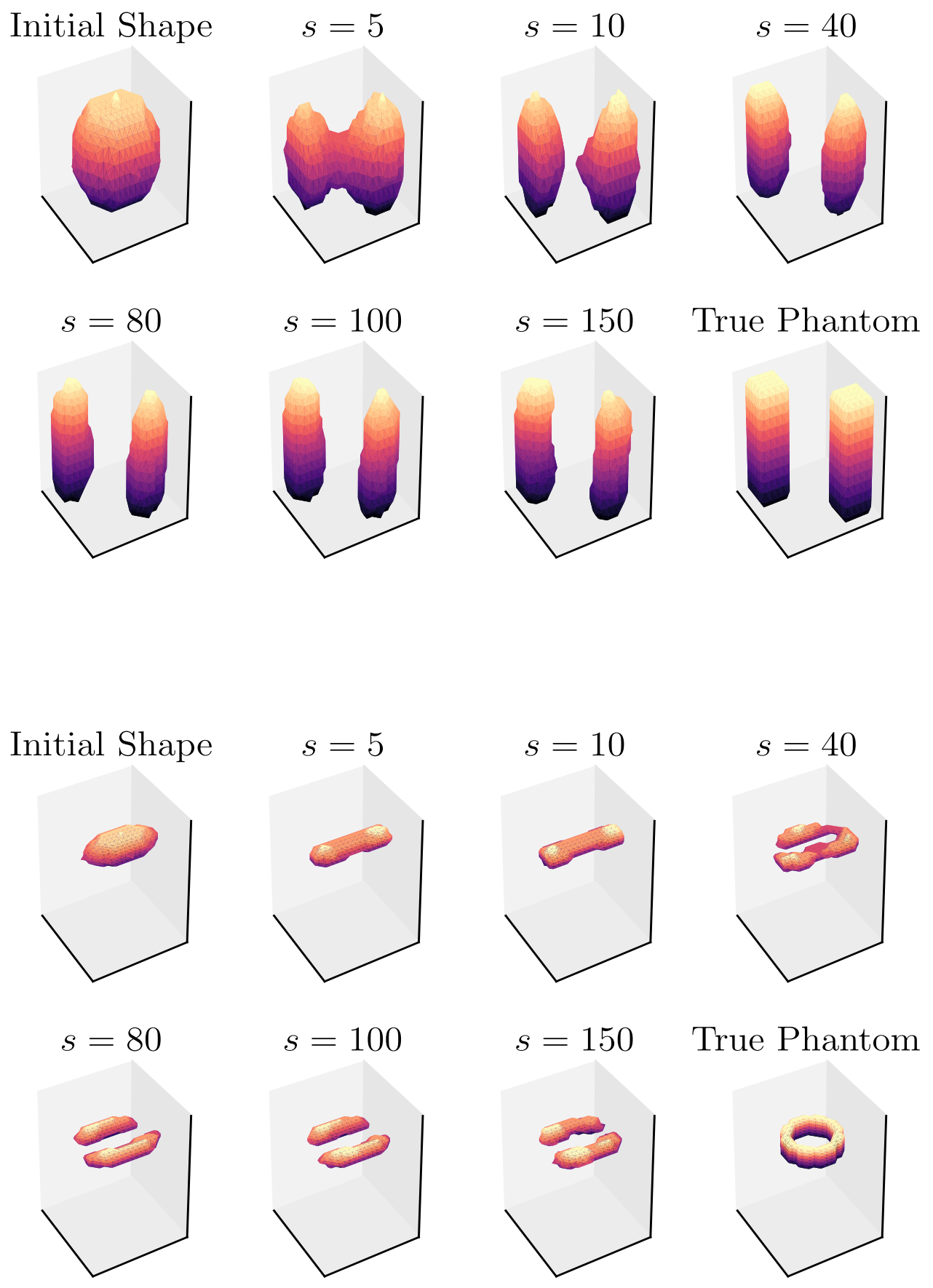

Figure 2. Surface plots of 3D shape evolution. Shown are (from left to right) the initial shape, snapshots at iteration numbers $s=5,10,40,80,100,150$, and the reference shape. Top two rows: test phantom 1; Bottom two rows: test phantom (2). 

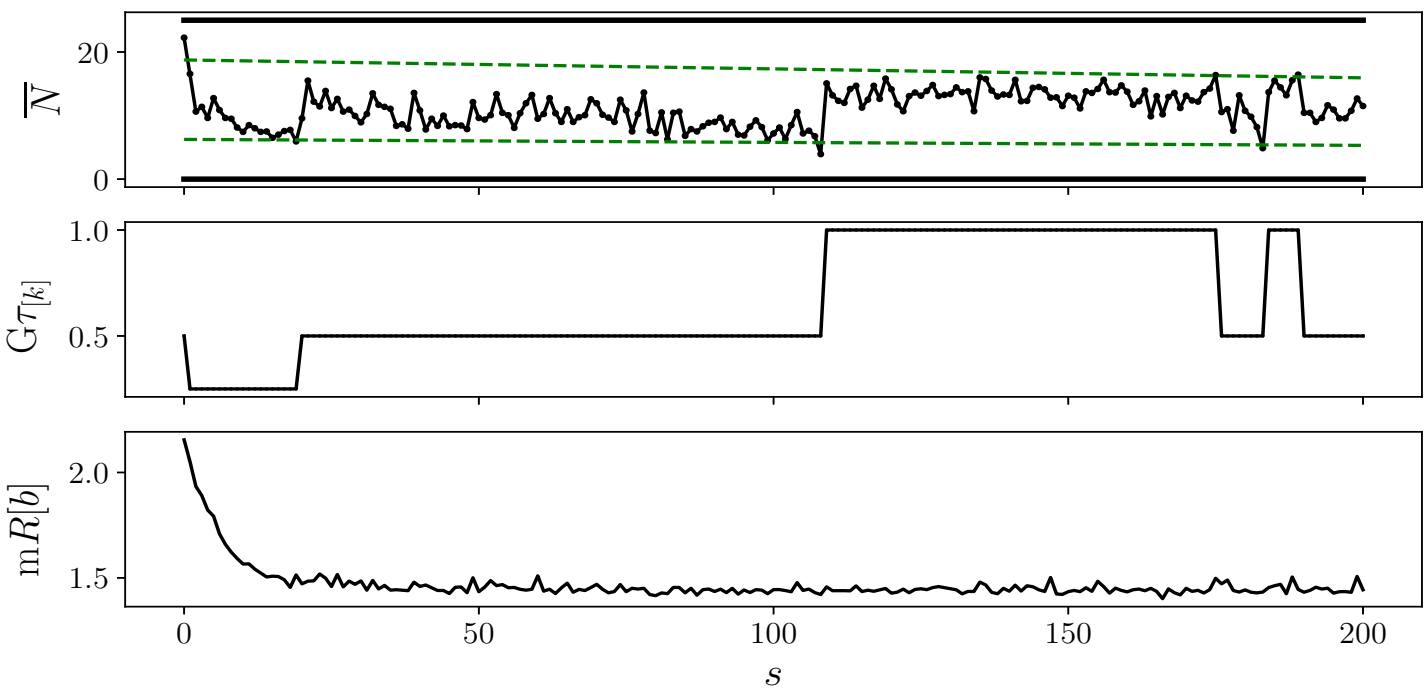

Figure 3. Evolution of line search parameters $\bar{N}, G \tau_{k}$ (Giga $\tau_{k}$ ) and of the pseudo residual $m R[b]$ (part per thousand) against iteration number for test phantom 1.

For computational purposes, we monitor the cost evolution per sweep number $s$ by using an approximate (pseudo or lagged) data misfit functional

$$
\hat{\mathcal{J}}[b]^{s}=\sum_{j=1}^{n_{s}} \mathcal{J}_{j}\left[b_{j}\right],
$$

where the right hand sum is taking the values recorded during sweep $s$ before an update is made, as we can compute those values 'on the fly' without significant computational effort. This seems justified since calculating the correct residuals by running $n_{s}$ additional forward problems (not shown here) demonstrated that the differences are minor and not significant for monitoring purposes. By observation of Figure 3, we see that the pseudo residual converges as the sweeps progress. Also shown is the dynamic updating of $\tau_{k}$ as LK-Level set attempts to retain $\bar{N} \in I_{\text {target }}^{\prime}$ each sweep.

\subsection{Level set reconstruction with incorrectly chosen contrast value}

As demonstrated in the first two numerical experiments described in Figures 2-3, the level set method performs reasonably well in recovery of location and shape, under the assumption that interior and exterior values of conductivity are known. We claimed earlier that for very low frequency situations an rough approximation to the internal conductivity value is sufficient in order to obtain reasonable shape reconstructions. This has been confirmed by various numerical experiments so far, of which we will show only one representative case in this section. Additional examples will be shown elsewhere. For this purpose, let us now consider a level set reconstruction where the conductivity value is assumed only half the correct value during the iterations. Figure 4 shows surface plots of the 3D LK-Level set algorithm under this incorrectly chosen interior value for 


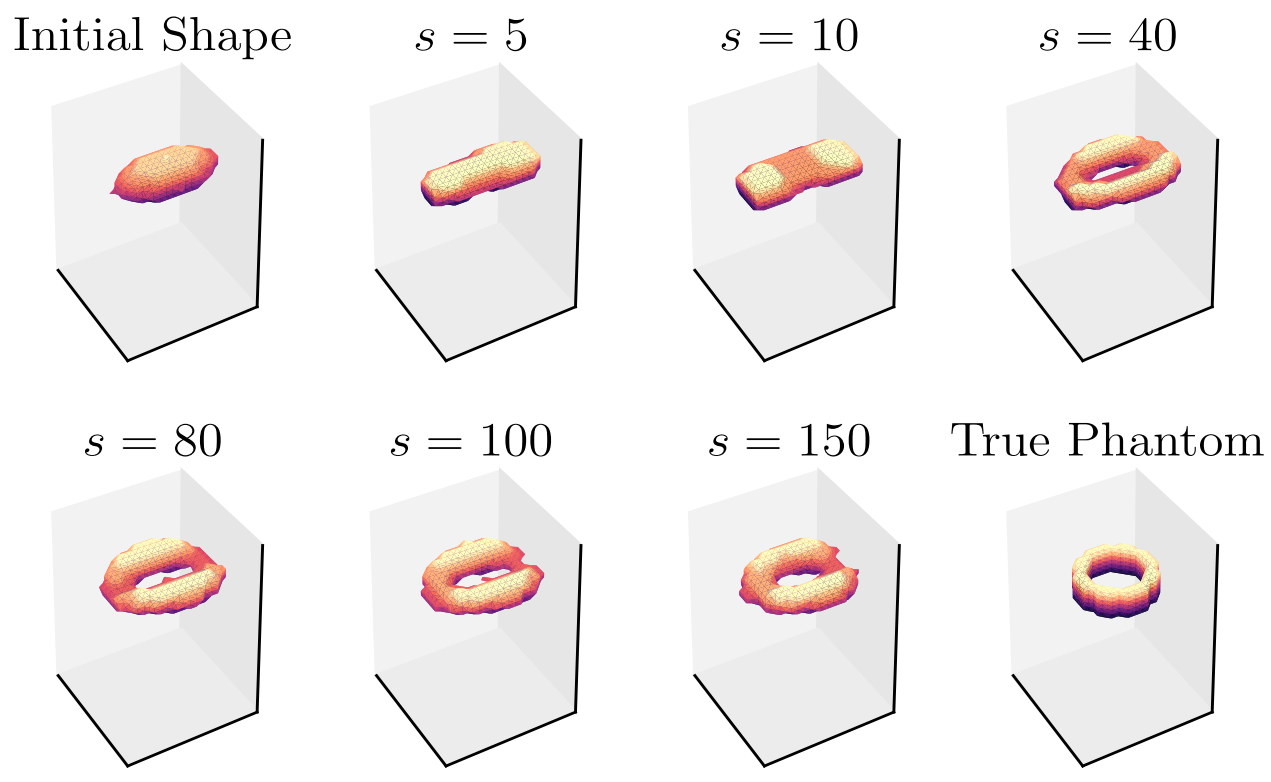

Figure 4. Surface plots of $3 \mathrm{D}$ shape evolution for test phantom 2. Here the internal conductivity value is assumed during the reconstruction $\sigma=0.25 \mathrm{~S} / \mathrm{m}$ instead of the correct value $\sigma=0.5 \mathrm{~S} / \mathrm{m}$. Shown are (from left to right) the initial shape, snapshots at iteration numbers $s=5,10,40,80,100,150$, and the reference shape.

test phantom (2). By observation we see that the algorithm responds to the modelling error of underestimating $b_{i}$ by recovering the inclusions with a similar shape as the correct one, but slightly 'inflated', i.e. covering a larger volume. This is still acceptable in situations where the main goal is to identify and locate certain target objects, such as threats hidden in boxes or containers or certain objects hidden in caves or rooms. We mention that similarly an overestimation of the internal conductivity by a factor or 2 , for example, reconstructs a shrunk version of the correct shape. Still, the true overall shape remains visible. Smaller deviations from the true internal value have minor effects on the reconstructions, and if needed an additional internal optimization loop over this internal value can be incorporated into the level set based reconstruction scheme. This internal optimization loop, however, tends to converge only very slowly towards the correct value and significantly slows down the overall reconstruction scheme, such that it is left out of the demonstrations in this paper.

\subsection{Sparsity regularized reconstruction and comparison of all three schemes}

Figure 5 compares different reconstructions with each other using Algorithm $1\left(L^{2}\right.$ based), Algorithm 2 (sparsity promoting with different sparsity levels) and Algorithm 3 (level set based with exact internal value) by using test phantom 1 . It shows in rows 
3-5 the same 2D cross-sections of different LK-Sparsity reconstructions of test phantom 1 and compares them with the level set (row 2) and $L^{2}$-based (row 6) counterparts. Row 1 of the image shows the reference profile. We note in the images of rows 3-5 that the gradient of the conductivity between neighbouring cells in non zero regions increases as one reduces the threshold parameter. The visual comparison with the level set based shape reconstruction in row 2 and the $L^{2}$ based voxel reconstruction shows that the sparsity reconstructions tend to provide some form of trade-off between compact reconstructions of the level set approach and the smooth profiles of $L^{2}$ based reconstructions, depending on the chosen sparsity level. This is to be expected, as the sparsity term can be considered a valid regularizer where the threshold value is part of the regularization scheme. However, insisting on a very sparse conductivity profile can allow the recovered shape to be distorted, with some parts of the object becoming suppressed by the algorithm, as can be seen in the reconstruction of the long bar on the right of the images. Therefore care must be taken when choosing the threshold parameter. The level set reconstruction does not a priori enforce sparsity in the voxel basis, such that extended objects tend to be better reconstructed by such a shape based approach compared to sparsity approaches.

Figure 6 shows further comparisons for test phantom 1. The top image compares the data misfit for different sparsity levels $\Lambda$ against sweep number. The center images compares the three reconstruction schemes with respect to their (pseudo) data misfits against sweep number. As we reduce the sparsity thresholding term, we see that the data misfit is shifted by a factor downward. In comparison with the reconstructions in Figure 5, this is consistent; since increasing the threshold yielded a reconstruction which has less resemblance with the true phantom. As we lower the threshold parameter, the cost curve shifts closer to the LK-Pixel cost. This is to be expected, as LK-Sparsity regularizes the LK-Pixel problem. Also, for giving a fair treatment to all three algorithms in this comparison, we need to keep in mind that we only plot the pseudo-data misfit here, which is not explicitly minimized in the sparsity promoting reconstruction scheme where an additional sparsity term appears in the cost functional. Finally, the bottom image of the figure shows a line plot of the diagonal in each reconstruction at the stated $z$ for test phantom 1. It highlights the behaviour of each reconstruction scheme compared to the true phantom. By observation of the line plots, we can see peaks in the same region as the true phantom across all three reconstruction schemes, with LK-Sparsity attempting to zero fill where there is no object. The smooth behaviour of the LK-Pixel scheme is also clear to see. 

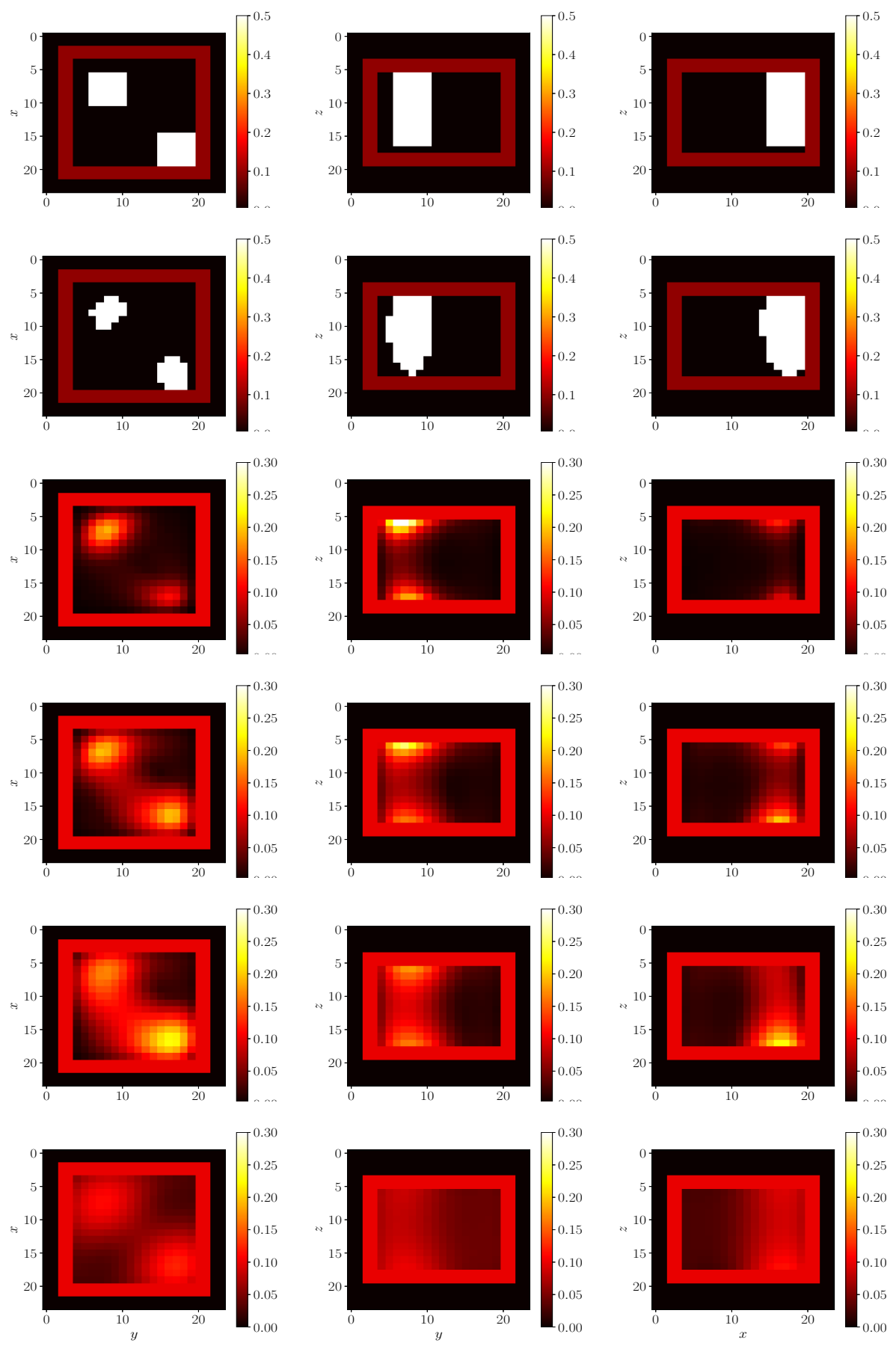

Figure 5. Top: Slices of test phantom 1 Left $z=44$, middle $x=17$, right $y=26$. First row: Reference medium; Second row: Level set based reconstruction; Third row: 3D LK-Sparsity reconstruction, $s=100$ and $\left(\tau, d_{1}\right)=\left(7.7413 \times 10^{5}, 0.95\right)$. Fourth row: 3D LK-Sparsity reconstruction, $s=100$ and $\left(\tau, d_{1}\right)=\left(7.7413 \times 10^{5}, 0.80\right)$. Fifth row: 3D LK-Sparsity reconstruction, $s=100$ and $\left(\tau, d_{1}\right)=\left(7.7413 \times 10^{5}, 0.60\right)$. Sixth row: 3D LK reconstruction without sparsity enforcement. 

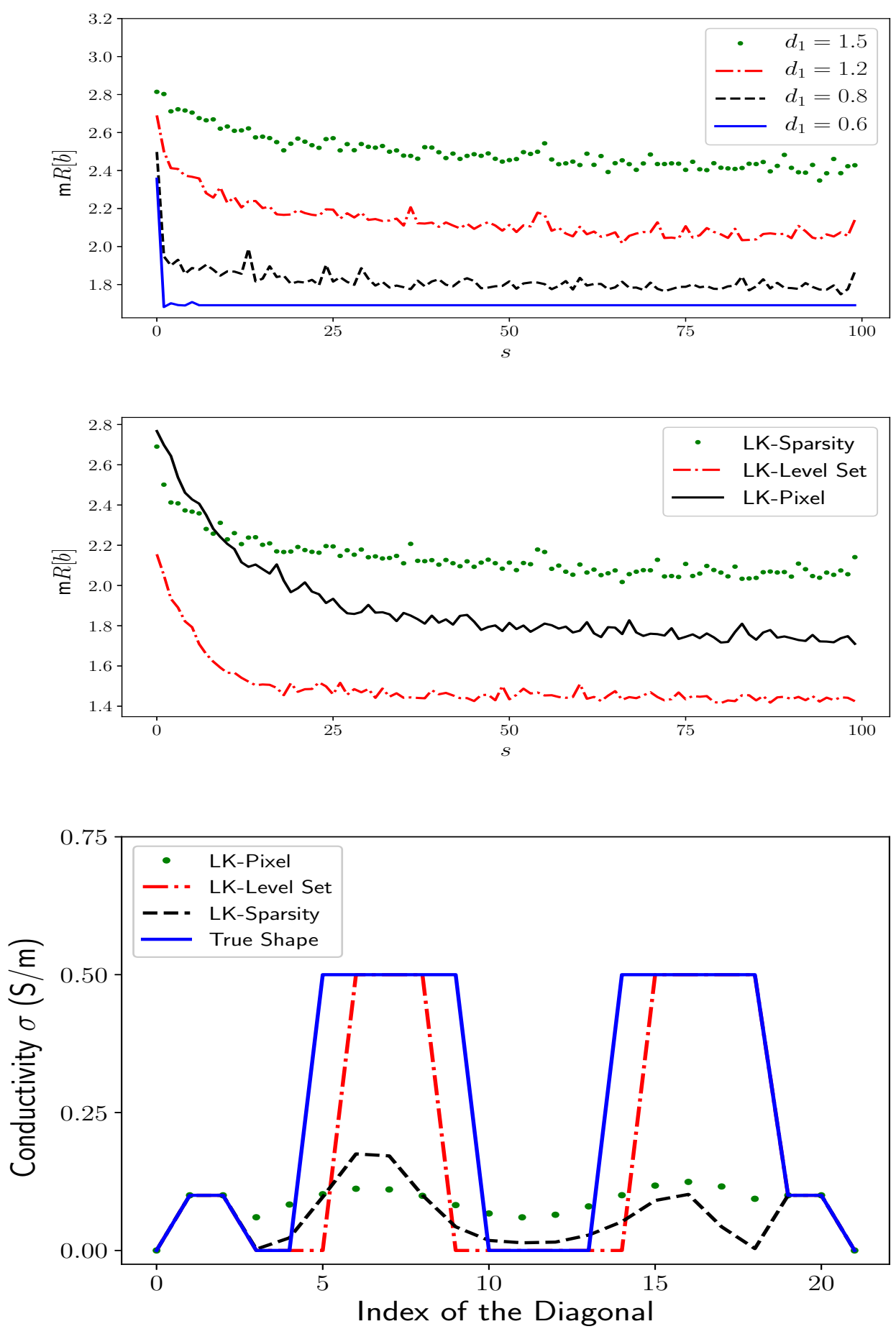

Figure 6. Top: Evolution of pseudo-cost for test phantom 1 with varied sparsity level $d_{1}$; Middle: Comparison of pseudo-cost across all three reconstruction schemes for test phantom 1 , with sparsity level $d_{1}=1.20$; Bottom: Line plots of the diagonal at $z=44$ for test phantom 1 , with sparsity level $d_{1}=1.2$ used. 


\section{Summary and Conclusion}

We have introduced two new reconstruction algorithms for the imaging of shielded containers using near-field electromagnetic data. A comparison of the performance for our two proposed reconstruction schemes was made with an established scheme and between each other. The first new algorithm solves a shape-based EM inverse problem by adapting general ideas described in $[18,22]$ for application in this new challenging situation. The second one solves a pixel-based EM inverse problem but promotes sparsity in the solution through regularization. The method used here follows that proposed in [50] (for a different 2D imaging modality) and extends it to 3D imaging from near-field electromagnetic data.

The performances of all three algorithms are promising for constructing a general purpose algorithm. However, when a priori knowledge is available, indicating that inclusions with small compact support and significantly higher internal conductivity value are present in the domain of interest, then both the sparsity regularized and the level set based shape evolution approach perform better than the standard $L^{2}$-based scheme. This is true even in situations where the $L^{2}$-scheme reduces the least squares data misfit functional to the same extent as the other two schemes. Due to the additional constraints included in the sparsity and level set based algorithms, reconstructions are more likely to yield results which are in agreement with such prior information.

The numerical experiments presented in this paper using noisy simulated data served as a proof-of-concept study, where it is useful to have full control over the noise level. In the future, we aim at investigating robustness of all three schemes with respect to independently generated data (using a completely different forward simulation tool, or ideally real data). In addition, we want to investigate the possibility of reconstructing more than one electromagnetic material parameter, as well as incorporating anisotropy in the parameter distributions. Furthermore, we want to increase the conductivity of embedded objects as well as our shielded walls in order to verify the limitations and capabilities of these algorithms in a variety of practically important applications. Finally, we will aim at applying these techniques to situations at a variety of scales, possibly including multi-frequency or broad-band electromagnetic data at suitable frequency ranges.

\section{Appendix A: An efficient line search strategy for Kaczmarz iterations}

In this Appendix, we propose a novel line-search strategy to be employed in the LK-Pixel and LK-Sparsity algorithms that are presented in this paper.

To start with, we consider a preprocessing step (involving a sufficiently large subset of the entire data set) which gives us a rough estimate of how $\tau_{0}$ should be chosen. Then, we dynamically update $\tau_{k}$ in the following sweeps using a different criteria. For this purpose, we choose an $n_{0}$ sufficiently large $\left(1 \leq n_{0} \leq n_{s}\right)$ and solve $n_{0}$ forward and $n_{0}$ adjoint problems for computing $\nabla \mathcal{J}_{n_{0}}\left[b_{0}\right]$ based on this subset of the data, where 
$\nabla \mathcal{J}_{n_{0}}\left[b_{0}\right]$ admits the decomposition: $\nabla \mathcal{J}_{n_{0}}\left[b_{0}\right]=\sum_{j \in\left\{j_{1}, j_{2}, \cdots, j_{n_{0}}\right\}} \nabla \mathcal{J}_{j}\left[b_{0}\right]$. Whereas the succeeding Kaczmarz type approach usually only uses the information of one single source position in one update, in this first step a subset of $n_{0}$ sources should be chosen to combine information of a significant subset of source positions for calculating a more reliable initial estimate of the gradient $\nabla \mathcal{J}\left[b_{0}\right]$. We usually would choose a quarter or half of all available sources, but we could use less or more than those if this seems reasonable. The choice of source indices can follow a random rule (as it is done here and is often done in stochastic gradient schemes [27]) or alternatively we can select source indices following a more systematic way. Once we have calculated $\nabla \mathcal{J}_{n_{0}}\left[b_{0}\right]$, we devise a method for computing $\tau_{0}$ as follows. Instead of considering the cost functional that incorporates the entire data set, we consider in our initial step a reduced cost functional $S_{0}$ which only considers the chosen subset of $n_{0}$ source positions. In a line search we will monitor its behaviour with respect to various choices of $\tau$

$$
\mathcal{S}_{0}(\tau)=\|\tilde{\mathcal{R}}[b(\tau)]\|_{L^{2}(\Omega)}^{2},
$$

where

$$
\tilde{\mathcal{R}}[b(\tau)]=\sum_{i \in\left\{j_{1}, j_{2}, \cdots, j_{n_{0}}\right\}} \mathcal{R}_{i}[b(\tau)] \quad \text { and } \quad b(\tau)=b_{0}-\tau \nabla \mathcal{J}_{n_{0}}\left[b_{0}\right] .
$$

We now follow a variant of the so-called Brent method [49], which we adjust to the Kaczmarz type scheme. Let us define $I_{\tau}=\left[\tau_{\min }, \tau_{\max }\right]$ and denote $\tau^{*} \in I_{\tau}$ the minimizer of $\mathcal{S}_{0}(\tau)$ (assuming that there is only one in the given interval). We choose here

$$
\tau_{\text {max }}=c_{1} \frac{\left\|b_{0}\right\|_{2}}{\left\|\nabla \mathcal{J}_{n_{0}}\left[b_{0}\right]\right\|_{2}}, \tau_{\text {min }}=c_{1} \tau_{\text {max }},
$$

where $c_{1} \in(0, a), a \in \mathbb{R}^{+}$, but other choices are possible. Typically, $a$ is chosen to be much less than unity so that we don't promote large $\tau$ which might cause problems in a Kaczmarz type scheme. Note that choosing $n=n_{s}$ retains our original cost functional and performs a traditional line search in this first step. In practice, a small fraction of $n_{s}$ sources seem sufficient for choosing $\tau_{0}$. And so, (21) can be evaluated for $\tau=\tau_{\text {min }}, \tau_{\text {mid }}, \tau_{\text {max }}$; where $\tau_{\text {mid }}$ is the middle point of the interval $I_{\tau}$.

Once the residual quantities have been computed, we choose

$$
\tau_{0}= \begin{cases}\tau_{\text {min }} & \text { if } \tilde{R}\left[b\left(\tau_{\text {min }}\right)\right]<\tilde{R}\left[b\left(\tau_{\text {mid }}\right)\right] \text { and } \tilde{R}\left[b\left(\tau_{\text {max }}\right)\right] ; \\ \tau_{\text {mid }}^{q} & \text { if } \tilde{R}\left[b\left(\tau_{\text {mid }}\right)\right]<\tilde{R}\left[b\left(\tau_{\text {min }}\right)\right] \text { and } \tilde{R}\left[b\left(\tau_{\text {max }}\right)\right] \text { where } \\ & \tau_{\text {mid }}^{q} \text { is the minimizer of a quadratic polynomial } \\ & \phi^{q}(\tau)=a \tau^{2}+b \tau+c \text { fitted to } \tau_{\text {min }}, \tau_{\text {mid }}, \tau_{\text {max }} \\ \tau_{\text {max }} & \text { if } \tilde{R}\left[b\left(\tau_{\text {max }}\right)\right]<\tilde{R}\left[b\left(\tau_{\text {min }}\right)\right] \text { and } \tilde{R}\left[b\left(\tau_{\text {mid }}\right)\right] .\end{cases}
$$

Once $\tau_{0}$ has been chosen, the algorithm described in (9) proceeds and $\tau_{k}$ is dynamically updated such that

$$
\tau_{k+1}= \begin{cases}\tau_{k} & \text { if } \mathcal{J}\left[b_{k}\right]-\mathcal{J}\left[b_{k-1}\right]<0 \\ \left(1-c_{1}\right) \tau_{k} & \text { if } \mathcal{J}\left[b_{k}\right]-\mathcal{J}\left[b_{k-1}\right]>0\end{cases}
$$

for $k \geq 1, \tau_{1}=\tau_{0}$ and $c_{1} \in[0.5,1)$. This dynamic correction helps the algorithm stabilize as it tries to stop it from overestimating. Practically, we approximate $\mathcal{J}\left[b_{k}\right]$; as is discussed in section 8 . 


\section{Appendix B: A new line search strategy for Kaczmarz guided level set evolution}

In this Appendix, we describe an improved line search strategy based on one previously presented in [21] for level set based shape evolution scheme in the Kaczmarz setting.

In shape evolution schemes, the overall progress of the inversion can be directly quantified by counting the number of voxels that change values from $b_{i}$ to $b_{e}$ (and vice versa), in each step of the inversion. Practically, this quantity resembles a shape velocity multiplied by the time step taken in each artificial evolution step; which is a reasonable quantity to control in such shape evolution approaches. When insisting on a smooth evolution of the shape across all individual Kaczmarz updates, we would hope that each update provides such a voxel count in a certain range of admissible values. Therefore, in order to maintain convergence of the algorithm, we record the number of voxels $N_{j}$ that change value in the $k$ th update. Our aim is to choose $\tau_{k}$ such that $N_{j}$ lies in an interval deemed suitable for the shape to evolve smoothly. The relationship between $N_{j}$ and the data misfit is currently unclear. However, we can follow standard ideas of a backtracking line search for $\tau_{k}$ such that $N_{j} \in I_{\text {target }}$ where $I_{\text {target }}=\left[N_{\text {inf }}, N_{\text {sup }}\right]$ is chosen appropriately. An insistence of $N_{j} \in I_{\text {target }}$ does not alleviate the problem of giving equal weighting to each sensitivity pattern of the $j$ th source. To tackle this, we employ a dynamic correction of $\tau_{k}$ each sweep. In particular, we compute the average number of voxels $\bar{N}=\left(1 / n_{s}\right) \sum_{j=1}^{n_{s}} N_{j}$ each sweep and try to adjust $\tau_{k}$ so $\bar{N} \in I_{\text {target }}^{\prime}=\left[N_{\text {low }}, N_{\text {high }}\right]$ such that

$$
N_{\text {inf }}<N_{\text {low }}<N_{\text {high }}<N_{\text {sup }}
$$

Hence

$$
\tau_{k}= \begin{cases}k_{1} \tau_{k-1} & \text { if } \bar{N}>N_{\text {high }} \text { where } k_{1} \in[0.5,1) ; \\ k_{2} \tau_{k-1} & \text { if } \bar{N}<N_{\text {low }} \text { where } k_{2} \in(1,2],\end{cases}
$$

where $k=i n_{s}$ for $i=1,2, \cdots, S$. For other $k$ (i.e. per source), we perform a backtracking line search where we hope $\tau_{k}$ is sufficiently large so that it must be reduced to ensure that $N_{j} \in I_{\text {target }}$. Note that we initialize $\tau_{0}$ such that $\bar{N} \in I_{\text {target }}$, as this alleviates the issue of choosing $\tau_{0}$ too small or large.

The exact relationship between the number of voxels that change and the residual is not directly monitored here (otherwise the scheme would become less efficient). Intuitively, one expects that $\bar{N}$ reduces over an algorithms lifespan; due to diminishing returns of gradient descent methods. An artificial way of addressing this is to introduce a dynamic interval $I_{\text {target }}=I_{\text {target }}(s)$, which induces $I_{\text {target }}^{\prime}=I_{\text {target }}^{\prime}(s)$. In particular, we insist that

$$
\begin{aligned}
& \hat{N}_{\text {high }}(s, \eta)=\frac{1}{S}\left(N_{\text {low }}-N_{\text {high }}\right) \eta s+N_{\text {high }} ; \\
& \hat{N}_{\text {low }}(s, \eta)=-\frac{1}{S} N_{\text {low }} \eta s+N_{\text {low }},
\end{aligned}
$$

where $\eta$ is a parameter that dictates 'shrinkage' of the admissible interval range $I_{\text {target }}$. Note that $\eta=0$ yields retention of the constant interval. 


\section{Acknowledgements}

The work of AH was supported by the Nuclear Threat Reduction Department of AWE, UK, as part of his Ph.D. project, under the Research Project Number 30400564.

\section{References}

[1] A. Abubakar and P.M. van den Berg. Iterative forward and inverse algorithms based on domain integral equations for three-dimensional electric and magnetic objects. J. Comput. Phys., 195:236-262, 2004.

[2] H.F. Alqadah, N.P. Valdivia, and E.G. Williams. A super-resolving near-field electromagnetic holographic method. IEEE Trans. Antennas and Propagation, 62(7):3679-3692, 2014.

[3] D.A. Aruliah. Fast solvers for time-harmonic Maxwell's equations in 3D. PhD thesis, University of British Columbia, 2001.

[4] D.A. Aruliah, U.M. Ascher, and D. Oldenburg. A method for the forward modelling of 3-d electromagnetic quasi-static problems. Mathematical Models and Methods in Applied Sciences, 11(1):1-21, 2001.

[5] A. Beck and M. Teboulle. A fast iterative schrinkage-thresholding algorithm for linear inverse problems. SIAM J. Imaging Sciences, 2(1):183-202, 2009.

[6] T. Bonesky, K. Bredies, D. Lorenz, and P. Maass. A generalized conditional gradient method for nonlinear operator equations with sparsity constraints. Inverse Problems, 23(5):2041-2058, 2007.

[7] L. Bottou, F.E. Curtis, and J Nocedal. Optimization methods for large scale machine learning. SIAM Review, 60(2):223-311, 2018.

[8] B.M. Brown, M. Marletta, and J.M. Reyes. Uniqueness for an inverse problem in electromagnetism with partial data. Journal of Differential Equations, 260:6525-6547, 2016.

[9] M. Burger. A level set method for inverse problems. Inverse Problems, 17(5):1327-1355, 2001.

[10] M. Burger and S. Osher. A survey on level set methods for inverse problems and optimal design. European J. Appl. Math., 16:263-301, 2005.

[11] P. Caro, P. Ola, and M. Salo. Inverse boundary value problem for maxwell equations with local data. Communications in Partial Differential Equations, 34(11):1425-1464, 2009.

[12] X. Chen. Computational Methods for Electromagnetic Inverse Scattering. IEEE Press. John Wiley \& Sons Singapore, 2018.

[13] D. Colton and R. Kress. Inverse Acoustic and Electromagnetic Scattering Theory, volume 93 of Applied Mathematical Sciences. Springer Science and Business Media, 3rd edition, 2013.

[14] B.J. Darrer. Electromagnetic induction imaging through metallic shields. PhD thesis, University College London, 2017.

[15] B.J. Darrer, J.C. Watson, P. Bartlett, and F. Renzoni. Magnetic imaging: a new tool for uk national nuclear security. Scientific reports, 5:7944, 2015.

[16] B.J. Darrer, J.C. Watson, P. Bartlett, and F. Renzoni. Toward an automated setup for magnetic induction tomography. IEEE Transactions on Magnetics, 51(1):1-4, 2015.

[17] I. Daubechies, M. Defrise, and C. De Mol. An iterative thresholding algorithm for linear inverse problems with a sparsity constraint. Communications on Pure and Applied Mathematics, 57(11):1413-1457, 2004.

[18] O. Dorn and U. Ascher. Shape reconstruction in 3d electromagnetic induction tomography using a level set technique. Proc. 23rd International Review of Progress in Applied Computational Electromagnetics ACES, pages 1-6, 2007.

[19] O. Dorn, H. Bertete-Aguirre, J.G. Berryman, and G.C. Papanicolaou. A nonlinear inversion method for 3d electromagnetic imaging using adjoint fields. Inverse problems, 15(6):1523, 1999.

[20] O. Dorn, H. Bertete-Aguirre, J.G. Berryman, and G.C. Papanicolaou. Sensitivity analysis of 
a nonlinear inversion method for $3 \mathrm{~d}$ electromagnetic imaging in anisotropic media. Inverse problems, 18(2):285, 2002.

[21] O. Dorn and A. Hiles. A level set method for magnetic induction tomography of 3d boxes and containers. In D. Lesselier and C. Reboud, editors, Electromagnetic Non-Destructive Evaluation (XXI), pages 33-40. IOS Press, 2018.

[22] O. Dorn and D. Lesselier. Level set methods for inverse scattering. Inverse Problems, 22:R67R131, 2006.

[23] O. Dorn and D. Lesselier. Level set methods for inverse scattering - some recent developments. Inverse Problems, 25:125001, 2009.

[24] O. Dorn, E. Miller, and C. Rappaport. A shape reconstruction method for electromagnetic tomography using adjoint fields and level sets. Inverse Problems, 16:492-506, 2000.

[25] S. Foucart and H. Rauhut. A mathematical introduction to compressive sensing. Applied and Numerical Harmonic Analysis. Birkhauser, 2013.

[26] P. González-Rodríguez, M. Kindelan, M. Moscoso, and O. Dorn. History matching problem in reservoir engineering using the propagation-backpropagation method. Inverse Problems, 21:565$590,2005$.

[27] I. Goodfellow, Y. Bengio, and A Courville. Deep Learning. The MIT Press, 2016.

[28] E. Haber. Quasi-newton methods for large scale elctromagnetic inverse problems. Inverse Problems, 21:305-323, 2005.

[29] E. Haber and U.M. Ascher. Fast finite volume simulation of 3d electromagnetic problems with highly discontinuous coefficients. SIAM Journal on Scientific Computing, 22(6):1943-1961, 2001.

[30] E. Haber, U.M. Ascher, D.A. Aruliah, and D.W. Oldenburg. Fast simulation of 3d electromagnetic problems using potentials. Journal of Computational Physics, 163(1):150-171, 2000.

[31] F. Hettlich. The domain derivative of time-harmonic electromagnetic waves at interfaces. Mathematical Methods in the Applied Sciences, 35:1681-1689, 2012.

[32] K. Ito, K. Kunisch, and Z. Li. Level-set function approach to an inverse interface problem. Inverse Problems, 17(5):1225-1242, 2001.

[33] B. Jin, T. Khan, and P. Maass. A reconstruction algorithm for electrical impedance tomography based on sparsity regularization. International Journal for Numerical Methods in Engineering, 89:337-353, 2012.

[34] B. Jin and P. Maass. Sparsity regularization for parameter identification problems. Inverse Problems, 28(12):123001, 2012.

[35] Q. Jin and W. Wang. Landweber iteration of kaczmarz type with general non-smooth convex penalty functional. Inverse Problems, 29(8):085001, 2013.

[36] B. Kaltenbacher, A. Neubauer, and O. Scherzer. Iterative Regularization Methods for Nonlinear Ill-Posed Problems. de Gruyter, 2008.

[37] T. Kluth. 3d electrical impedance tomography with sparsity constraints: Algorithm and implementation in application to the complete electrode model. Master's thesis, University of Bremen, 2011.

[38] A. Leitão and M. Marques Alves. On Landweber-Kaczmarz methods for regularizing systems of ill-posed equations in Banach spaces. Inverse Problems, 28:104008(15pp), 2012.

[39] A. Leitão and O. Scherzer. On the relation between constraint regularization, level sets, and shape optimization. Inverse Problems, 19(1):L1-L11, 2003.

[40] A. Litman, D. Lesselier, and D. Santosa. Reconstruction of a two-dimensional binary obstacle by controlled evolution of a level-set. Inverse Problems, 14:485-706, 1998.

[41] L. Ma and M. Soleimani. Magnetic induction tomography methods and applications: a review. Meas. Sci. Technol., 28(072001), 2017.

[42] F. Margotti, A. Rieder, and A. Leitão. A kaczmarz version of the reginn-landweber iteration for ill-posed problems in banach spaces. SIAM J. Numer. Anal., 52(3):1439-1465, 2014.

[43] P.R. McGillivray, D.W. Oldenburg, R.G. Ellis, and T.M. Habashy. Calculation of sensitivities for 
the frequency-domain electromagnetic problem. Geophys.J.Int., 116:1-4, 1994.

[44] F. Natterer and F. Wuebbeling. Mathematical Methods in Image Reconstruction. Mathematical Modeling and Computation Series. SIAM, 2001.

[45] J.W. Neuberger. Sobolev Gradients and Differential Equations. Springer-Verlag, 1997.

[46] G.A. Newman and D.L. Alumbaugh. Three-dimensional massively parallel electromagnetic inversion-1. theory. Geophys.J.Int., 128:345-354, 1997.

[47] J. Nocedal and S.J. Wright. Numerical Optimization. Springer Science and Business Media, 2 edition, 2006.

[48] S. Osher and R. Fedkiw. Level set Method and Dynamic Implicit Surfaces, volume 153 of Applied Mathematical Sciences. Springer, 2003.

[49] W.H. Press, B.P. Flannery, S.A. Teukolsky, and W.T. Vetterling. Numerical Recipes in C. Cambridge University Press, 1988.

[50] K. Prieto and O. Dorn. Sparsity and level set regularization for diffuse optical tomography using a transport model in 2d. Inverse Problems, 33(1):014001, 2016.

[51] S. Sainson. Electromagnetic Seabed Logging: A new tool for geoscientists. Springer International Publishing Switzerland, 2017.

[52] F. Santosa. A level-set approach for inverse problems involving obstacles. ESAIM:Control, Optimization and Calculus of Variations, 1:17-33, 1996.

[53] H.G. Schantz, C. Weil, and A.H. Unden. Characterization of error in a near-field electromagnetic ranging (nfer) real-time location system (rtls). In Radio and Wireless Symposium (RWS), Phoenix, Arizona, pages 379-382. IEEE, 12011.

[54] J. Sethian. Level set Methods and Fast Marching Methods: Evolving Interfaces in Computational Geometry, Fluid Mechanics, Computers Visions and Material Sciences. Cambridge Monographs on Applied and Computational Mathematics. Cambridge University Press, 1996.

[55] M. Soleimani, W.R.B. Lionheart, and O. Dorn. Level set reconstruction of conductivity and permittivity from boundary electrical measurements using experimental data. Inverse Problems in Science and Engineering, 14(2):193-210, 2006.

[56] E. Somersalo, D. Isaacson, and M. Cheney. A linearized inverse boundary value problem for maxwell's equations. Journal of Computational and Applied Mathematics, 42:123-136, 1992.

[57] N.P. Valvidia and E.G. Williams. The reconstruction of surface tangential components of the electromagnetic field from near-field measurements. Inverse Problems, 23:785-798, 2007.

[58] P. Wang, Z. Liu, X. Zhang, L. Xu, J. He, and Y. Wan. Wideband signal based near-field electromagnetic ranging for indoor localization. In Advances in Intelligent Systems Research (AISR), 2018 International Conference on Advanced Control, Automation and Artificial Intelligence (ACAAI 2018), volume 155, pages 243-247, 2018.

[59] J. Wood, R. Ward, Ch. Lloyd, P. Tatum, C. Shenton-Taylor, S. Taylor, G. Bagley, M. Joseph, and J.C. Watson. Effect of shielding conductivity on magnetic induction tomographic security imagery. IEEE Transactions on Magnetics, 53(4):1-6, 2017.

[60] M.S. Zhdanov. Inverse Theory and Applications in Geophysics. Cambridge Monographs on Applied and Computational Mathematics. Elsevier, 2nd edition, 2015. 